\title{
Aesthetics in Orthodontics: Where We Are? Vincenzo Grassia*
}

Received: November 13, 2018; Accepted: November 15, 2018; Published: November 21, 2018

In our days the importance given by society and customs to aesthetics is always increasing. This obviously had important fallout also in the orthodontic field. More scientific works focus their attention on new aesthetic parameters that adapt to changes in the society in which we live. Requirements to achieve a better smile aesthetics increases continuously and at same time new tools with a low aesthetic impact were developed. Moreover, producers of orthodontic appliances are increasingly their research on products competitive for their effectiveness and predictability but also for their aesthetic and comfort features. An important upsurge of clear aligners and lingual orthodontics as well as other "invisible" techniques has been described in the orthodontic world. For the researchers, therefore, new objectives appear. The inclination to use these aesthetic devices is not reducing the level of quality in the results of orthodontic treatments? Are the objectives of orthodontic treatment not the functional restoration of the occlusion but only the aesthetics of the smile? that, although important, should not overshadow important aspects such as occlusal stability and functional balance. Therefore, the "social six" is certainly an approach that has always been considered valid in cases where the patient's "chief complaint" does not predispose at longer, more complex, invasive and often more expensive treatments, oriented for a rapid and contained resolution treatment of the six anterior superior teeth. However, this approach should always be considered the last treatment alternative, after the patient has been exhaustively educated on all treatment alternatives and their advantages and disadvantages. Often it could need avoided that the tool becomes the priority often with the disadvantage of the lower quality of final outcomes. Moreover, although clear aligners treatments have made important advances in the effectiveness and predictability of the movements, they are not yet comparable to the multibracket fixed appliances. Clear aligner treatments are a valid alternative only in cases where the effectiveness and efficacy of these aesthetic devices can be overlapped with just evidence based treatment.

Moreover, nowadays, the aesthetic demands of the patients become more complex and composite. Often, only one practitioner such as the orthodontist is not enough to satisfy all patient expectations. Teamwork is progressively essential, it need that different doctor specialist in the field of dentistry and medicine co-working. Indeed, the partnership with conservative dentists, prosthetists, periodontists, expand the prospective

\author{
Department of Orthodontics, Second \\ University of Naples, Italy
}

\section{*Corresponding author: Vincenzo Grassia, MDD, PhD \\ grassiavincenzo@libero.it}

\begin{abstract}
Multidisciplinary Department of MedicalSurgical and Dental Specialties, University of Campania "Luigi Vanvitelli", Via L. de Crecchio, 16-80138 - Naples, Italy.
\end{abstract}

Citation: Grassia V (2018) Aesthetics in Orthodontics: Where We Are? J Orthod Endod Vol.4 No.3:12

of an aesthetic treatment overcoming the limits of the single discipline. Collaborations with dental professionals and other medical-surgeons are also increasingly successful. An example is the growing numbers of patients who choose orthognathicsurgery treatments to improve facial aesthetics. These treatments require a close collaboration between the orthodontist and the maxillofacial surgeon who must cooperate synergistically for a common objective. The role of plastic surgeons and aesthetic doctors associated with the orthodontist is also increasing to ensure a complete approach to the aesthetic treatment of patients. Teamwork, however, requires special skills such as the ability to relate with different professional figures, training in areas not strictly their own. The final goal is to create a team qualified to provide facial and smile aesthetics at $360^{\circ}$. Surely it not due to underestimate the psychological component of the patients strongly motivated to change the aesthetics of the smile and face. Often these requests hide small or big relational problems that any aesthetic treatment can solve but only the competence of a psychiatrist and psychologist can treat. However, it is important to immediately intercept these kinds of problems and then direct them to the specific specialist.

It is, therefore, important nowadays to indulge the increasing aesthetic needs of patients but without leaving the basic aspects of orthodontic treatment. We have to refer constantly to the literature and we must not forget that also in the field of aesthetics the best possible approach is the evidence based one. Surely it is necessary to increase studies and research on new aesthetic tools and methods to highlight their limitations and advantages. 